\section{Guiding New Generations for Decades}

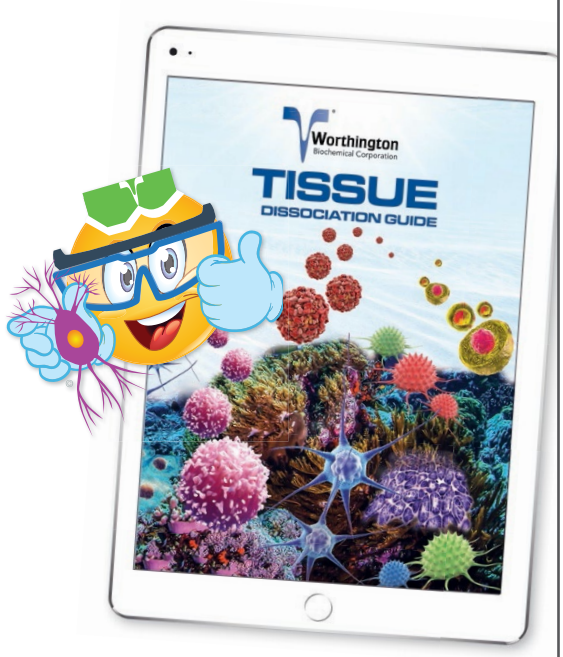

Worthington is supporting life science research and discovery, and we have done so for over 70 years.

Authoring technical manuals and guides both in print and digital formats. And, we are cited in thousands of respected scientific journals across the globe.

\section{Sharing Our Enzyme Expertise}

Order your free copy of the NEW Tissue Dissociation Guide, 18th Edition. Simply go to:

\section{Worthington-Biochem.com/ TissueDissociation/default.html}

\section{Come see us at AACO 19} Booth 2542

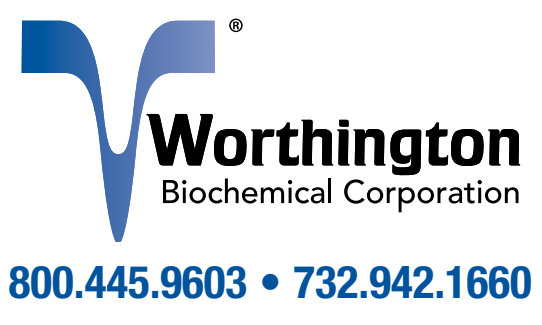

\title{
Atomic-level in-cell protein NMR
}

\author{
A newly developed method allows structure elucidation of proteins inside \\ intact mammalian cells, using solid-state NMR spectroscopy.
}

$\Lambda$ major challenge in structural biology is finding ways to observe biomolecules in their natural state. The complex environment and high viscosity of cells provide certain restraints over molecular functions that cannot be realistically re-created. This unique environment also leads to experimental limitations; for example, conventional solution-state NMR spectroscopy requires molecules to tumble fast in solution-a criterion that is difficult to meet within cells.

The research groups of Marc Baldus from Utrecht University and Huib Ovaa from Leiden University Medical Center (LUMC) have recently succeeded in using solid-state NMR spectroscopy (ssNMR) to investigate proteins inside human cells.

The method development came with several challenges. To be detected among all the other molecules in the cell, the molecule of interest needs to be isotopically labeled in isolation. Additionally, the ssNMR protocol needs to be sensitive enough to capture signals at endogenous protein concentrations in order for regular cell function to be maintained. Dynamic nuclear polarization (DNP) is a technique in which radicals containing unpaired electrons are used to polarize the molecule of interest, thereby enhancing the ssNMR sensitivity by more than a factor of 100 . However, the reducing environment inside cells makes using DNP for in-cell protein studies quite challenging.

The first challenge was easier to overcome: isotopically labeled protein was delivered to human cells by electroporation. The researchers note that other techniques, such as the use of cellpenetrating peptides, pore-forming toxins, or directed protein expression, may also be used. The signal enhancement was trickier. Baldus explains how they worked around the issue: "We noticed that the radicals actually diffused pretty fast within cells, and realized that we only needed 15 minutes for the DNP radicals to diffuse throughout the cell. The obvious solution was to add the polarizers at the end of the experiment, right before we do the NMR. When you measure this sample you have the protein where you want it to be, you have the sensitivity that you need, and you also have a fully functional protein."

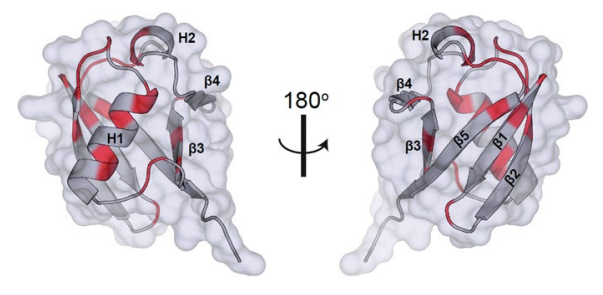

Structure of ubiquitin with unambiguously identified residues (shown in red). Reproduced from Narasimhan et al. (2019), Wiley-VCH Verlag.

The approach was demonstrated on ubiquitin, an important player in the protein-degradation machinery that has remained largely elusive to in-cell solutionstate NMR spectroscopy. Using 2D and 3D spectra for the labeled protein, they were able to obtain unambiguous signals for 25 out of 76 amino acids. Ovaa was excited about the results, emphasizing that "for the first time, we can look into cellular processes with atomic precision, without having to lyse the cells first."

The full potential of the method is still being explored, with several other, larger systems under scrutiny. Baldus adds, "DNP is a recent technology and currently available in about 20 labs around the world. To do such experiments, one needs cutting-edge NMR spectroscopy, as well as advanced biochemistry and molecular cell biology techniques-a combination that comes out of team effort." The two labs worked closely, particularly $\mathrm{PhD}$ students Siddarth Narasimhan and Alessandra Lucini Paioni at Utrecht, as well as Stephan Scherpe at LUMC, to design and optimize the technique.

With sophisticated in-cell methods in place, it will be very exciting to be able to observe subtle structural differences in proteins when they are bound to different partners.

\section{Arunima Singh}

Published online: 30 July 2019

https://doi.org/10.1038/s41592-019-0525-5

Research papers

Narasimhan, S. et al. DNP supported solid-state NMR of proteins inside mammalian cells. Angew. Chem. Int. Ed. Engl. https://doi.org/10.1002/anie.201903246 (2019). 